\title{
Time-Independent Reliability Analysis of Bridge System Based on Mixed Copula Models
}

\author{
Yuefei Liu ${ }^{1,2}$ and Xueping Fan ${ }^{1,2}$ \\ ${ }^{1}$ Key Laboratory of Mechanics on Disaster and Environment in Western China, Lanzhou University, \\ The Ministry of Education of China, Lanzhou, Gansu 730000, China \\ ${ }^{2}$ School of Civil Engineering and Mechanics, Lanzhou University, Lanzhou 730000, China \\ Correspondence should be addressed to Yuefei Liu; luiyuefei@126.com and Xueping Fan; fanxp@lzu.edu.cn
}

Received 25 December 2015; Accepted 19 May 2016

Academic Editor: Egidijus R. Vaidogas

Copyright (C) 2016 Y. Liu and X. Fan. This is an open access article distributed under the Creative Commons Attribution License, which permits unrestricted use, distribution, and reproduction in any medium, provided the original work is properly cited.

\begin{abstract}
The actual structural systems have many failure modes. Due to the same random sources owned by the performance functions of these failure modes, there usually exist some nonlinear correlations between the various failure modes. How to handle the nonlinear correlations is one of the main scientific problems in the field of structural system reliability. In this paper, for the two-component systems and multiple-component systems with multiple failure modes, the mixed copula models for time-independent reliability analysis of series systems, parallel systems, series-parallel systems, and parallel-series systems are presented. These obtained mixed copula models, considering the nonlinear correlation between failure modes, are obtained with the chosen optimal copula functions with the Bayesian selection criteria and Monte Carlo Sampling (MCS) method. And a numerical example is provided to illustrate the feasibility and application of the built mixed models for structural system reliability.
\end{abstract}

\section{Introduction}

Today's structural systems are becoming more complex and more sophisticated. Therefore, the evaluation of structural system reliability is becoming harder. This means that the derivations based on classical assumptions are no longer satisfactory for the analysis of systems in terms of reliability. The evaluation of today's real life systems needs more detailed and complicated statistical analysis. Dependence between the components is one of the intractable realistic assumptions that need to be carefully considered [1-4].

Especially for the actual bridge system, there exist many failure modes such as flexural failure and shearing failure. As the limit state functions (performance function) of these failure modes may have the same random sources, it is not mutually exclusive yet among failure modes [5-7]. Naturally, how to model the correlation among failure modes in aspect of the reliability analysis of structural systems is one of the most significant topics. The classic Pearson correlation coefficient is mainly used for characterizing the correlation among failure modes of structural system before; however, it has a few disadvantages. The copulas, unlike the Pearson correlation coefficient only applied for describing linear correlation, offer a flexible tool for deriving nonlinear dependence, especially tail dependence among failure modes. The aim of this study is to introduce the copula function as a useful tool for modeling the dependence among failure modes of bridge system. More recently, the copula theory has been primarily used in mechanical engineering [8] and hydraulic engineering [9] but little used in bridge engineering.

In this paper, firstly, several commonly used elliptical copulas and Archimedean copulas were introduced, and their application in the correlation analysis was also described in detail. And then based on the introduced copulas, with the aid of copula Bayesian selection criteria [7, 10, 11], a flexible mixed copula model is constructed, by means of linear weighted model, to model the nonlinear dependence among failure modes. For the two-component systems and multiple-component systems with multiple failure modes, the performance function value of failure modes is chosen as the copula functions' analytical variable to construct mixed copula model. With Monte Carlo Sampling method, the 
unknown parameters of copula functions can be approximately determined; therefore, the mixed copula function, considering the correlation between failure modes, is built. And then use the built mixed copula model to solve the structural system's reliability. Finally, a numerical example is provided to illustrate the feasibility and application of the built mixed copula models.

\section{Mixed Copula Models}

The mixed copula models are commonly built through different combinations of several copula functions, which respectively possess various characteristics in aspect of the dependence modeling. For the complex and ever-changing correlations among random variables, they are not adequate for modeling the dependence by means of only a single copula function. Therefore, it is necessary to build a flexible mixed copula model to describe the complex dependence structures. Moreover, it is essential to select several appropriate copula functions from the existing copulas before building the mixed copula model. The Bayesian selection criteria are just chosen for constructing the mixed copula model, as this method is independent of the parameter estimation and may be applied to all known copula classes $[7,10]$.

2.1. Basic Theory about Copula Functions. Sklar's theory [12] clearly indicated that, given random variables $\mathbf{X}=$ $\left(X_{1}, X_{2}, \ldots, X_{n}\right)$ with continuous marginal distribution functions $F_{X_{1}}, F_{X_{2}}, \ldots, F_{X_{n}}$ and $n$-dimensional joint distribution function $F$, there exists a unique copula function $C$ such that, for all $\mathbf{x}=\left(x_{1}, x_{2}, \ldots, x_{n}\right) \in \mathbf{R}^{n}$,

$$
\begin{aligned}
& F\left(x_{1}, x_{2}, \ldots, x_{n}\right) \\
& \quad=C\left(F_{X_{1}}\left(x_{1}\right), F_{X_{2}}\left(x_{2}\right), \ldots, F_{X_{n}}\left(x_{n}\right)\right) .
\end{aligned}
$$

According to (1), the joint probability density function of the random variable $\mathbf{X}=\left(X_{1}, X_{2}, \ldots, X_{n}\right)$ is given as follows:

$$
\begin{aligned}
& p\left(x_{1}, x_{2}, \ldots, x_{n}\right) \\
& \quad=c\left(F_{X_{1}}\left(x_{1}\right), F_{X_{2}}\left(x_{2}\right), \ldots, F_{X_{n}}\left(x_{n}\right)\right) \prod_{i=1}^{n} p_{X_{i}}\left(x_{i}\right),
\end{aligned}
$$

where $p_{X_{i}}\left(x_{i}\right)$ is the marginal probability density function (PDF) and $c(\mathbf{u})$ is the joint PDF of the copula function denoted as follows:

$$
c\left(u_{1}, \ldots, u_{n}\right)=\frac{\partial^{n} C}{\partial u_{1} \cdots \partial u_{n}}\left(u_{1}, \ldots, u_{n}\right) .
$$

If $F_{X_{i}}$ for $i=1,2, \ldots, n$ have generalized inverse functions $F_{X_{i}}^{-1}$ for $i=1,2, \ldots, n$, then, (1) can be rewritten as follows:

$$
\begin{aligned}
& F\left(F_{X_{1}}^{-1}\left(u_{1}\right), F_{X_{2}}^{-1}\left(u_{2}\right), \ldots, F_{X_{n}}^{-1}\left(u_{n}\right)\right) \\
& \quad=C\left(u_{1}, u_{2}, \ldots, u_{n}\right)
\end{aligned}
$$

where $u_{i}=F_{X_{i}}(i=1,2, \ldots, n),\left(u_{1}, u_{2}, \ldots, u_{n}\right)=\mathbf{u}$.
Equation (4) reveals how to construct the copula function of a multivariate distribution with given marginal distributions. It follows from the probability integral transform that the random variables $U_{i} \equiv F_{X_{i}}\left(X_{i}\right), i=1,2, \ldots, n$, are all uniformly distributed on $(0,1)$. Conversely, $X_{i}=F_{X_{i}}^{-1}\left(U_{i}\right), i=$ $1,2, \ldots, n, \mathbf{U}=\left(U_{1}, U_{2}, \ldots, U_{n}\right)$; with this in mind, (4) is expressed as

$$
\begin{aligned}
P & \left(X_{1} \leq x_{1}, X_{2} \leq x_{2}, \ldots, X_{n} \leq x_{n}\right)=P\left(X_{1}\right. \\
& \left.\leq F_{X_{1}}^{-1}\left(u_{1}\right), X_{2} \leq F_{X_{2}}^{-1}\left(u_{2}\right), \ldots, X_{n} \leq F_{X_{n}}^{-1}\left(u_{n}\right)\right) \\
& =P\left(U_{1} \leq u_{1}, U_{2} \leq u_{2}, \ldots, U_{n} \leq u_{n}\right) \\
& =C\left(u_{1}, u_{2}, \ldots, u_{n}\right) .
\end{aligned}
$$

According to (1)-(5), the copula function organically combines each marginal distribution function with the multivariate joint distribution functions; therefore, it not only considers the dependence among random variables but also simplifies the probability modeling process for multivariate random variables.

2.2. Bayesian Selection Criteria of Copula Functions. Huard et al. [10] proposed the Bayesian copula selection method applied to the bivariate copula functions. Bayesian copula selection method was deduced by the Bayesian hypothesis testing to choose the best copula. However, we apply this method to choose not just the best one but also the better copulas as the candidate copulas for constructing the mixed copula model.

For the Bayesian copula selection criteria, it is essential to propose the optical copula function selection criteria described by

$$
C_{\text {opt }}=\arg _{C_{l}} \max P\left(\mathrm{H}_{t} \mid D, I\right)
$$

where $C_{\text {opt }}$ is the optical copula function, $\mathrm{H}_{t}$ is denoted as the hypotheses that the right copula is copula $C_{l}(l=$ $1,2, \ldots, L)$, as the candidate copulas, $C_{l}$ is denoted by $C_{l}=$ $C_{l}\left(u_{i}, v_{i} ; \theta_{i}\right)(i=1,2, \ldots, n)$, and $n$ pair of quantiles $\left(u_{i}, v_{i}\right)$ are supposed as $n$ mutually independent pairs composing the data set $D$. Besides, $I$ stands for some a priori information. According to Bayesian formula, it can be obtained that

$$
P\left(\mathrm{H}_{t} \mid D, I\right)=\frac{P\left(D \mid \mathrm{H}_{t}, I\right) P\left(\mathrm{H}_{t} \mid I\right)}{P(D \mid I)},
$$

where $P\left(D \mid \mathrm{H}_{t}, I\right)$ is the likelihood, $P\left(\mathrm{H}_{t} \mid I\right)$ is the a priori copula family, and $P(D \mid I)$ is the normalization constant. The rank correlation coefficient Kendall's $\tau$, denoted by $\tau=g_{l}(\theta)$ to be the correlation parameters for all the selected copulas, is introduced in order to give the formula for $P\left(\mathrm{H}_{t} \mid D, I\right)$ :

$$
\begin{aligned}
P & \left(\mathrm{H}_{t} \mid D, I\right) \\
\quad & \int_{\tau \in \Omega} \frac{P\left(D \mid \mathrm{H}_{t}, \tau, I\right) P\left(\mathrm{H}_{t} \mid \tau, I\right) P(\tau \mid I)}{P(D \mid I)} d \tau,
\end{aligned}
$$



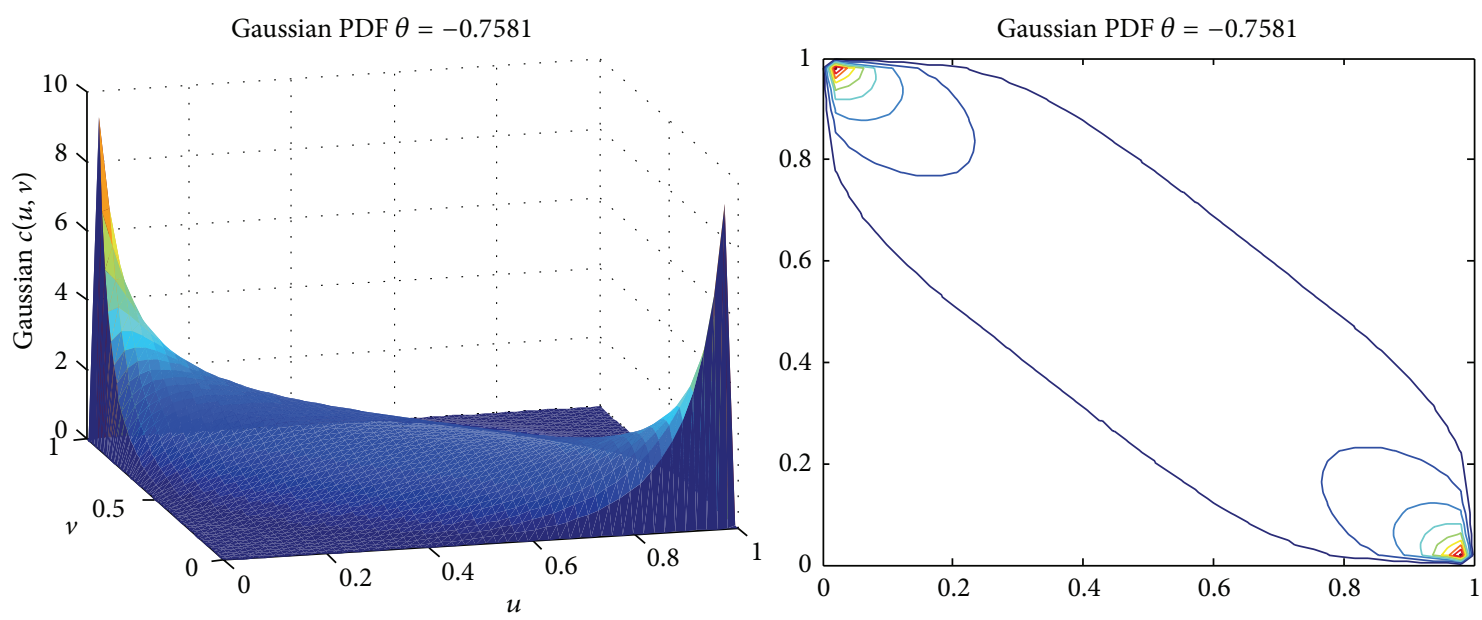

FIgURE 1: PDF and contour plots of Gaussian copula function.

where $P\left(D \mid \mathrm{H}_{t}, \tau, I\right)$ is the likelihood with respect to $\tau$, because of the $n$ mutually independent pairs, and is also described as the copula density:

$$
P\left(D \mid \mathrm{H}_{t}, \tau, I\right)=\prod_{l=1}^{n} C_{l}\left(u_{i}, v_{i} ; g_{l}^{-1}(\tau)\right) .
$$

Substituting (9) into (8), we obtain

$$
\begin{aligned}
& P\left(\mathrm{H}_{t} \mid D, I\right)=\frac{1}{P(D \mid I)} \int_{\tau \in \Omega} \prod_{l=1}^{n} C_{l}\left(u_{i}, v_{i} ; g_{l}^{-1}(\tau)\right) \\
& \quad P\left(\mathrm{H}_{t} \mid \tau, I\right) P(\tau \mid I) d \tau,
\end{aligned}
$$

where $P(D \mid I)$ is the normalization constant and $P\left(\mathrm{H}_{t} \mid\right.$ $\tau, I) P(\tau \mid I)=1 / \lambda(\Lambda)$, where $\lambda(\Lambda)$ is denoted as the Lebesgue measure of $\Lambda=[-1,1]$ in this paper; therefore, (10) can be also expressed as follows:

$$
P\left(\mathrm{H}_{t} \mid D, I\right) \propto \frac{1}{\lambda(\Lambda)} \int_{\Omega_{l} \cap \Lambda} \prod_{l=1}^{n} C_{l}\left(u_{i}, v_{i} ; g_{l}^{-1}(\tau)\right) d \tau .
$$

Obviously, the optimal copula function selection criteria can be described as the computation of the highest weight $W_{l}$ :

$$
W_{l}=\frac{1}{\lambda(\Lambda)} \int_{\Omega_{l} \cap \Lambda} \prod_{i=1}^{n} c_{l}\left(u_{i}, v_{i} ; g_{l}^{-1}(\tau)\right) d \tau
$$

In especial, the "right" copulas include not just the best copula with the highest weight but also the better copula with higher weight for the mixed copula modeling in this paper.

Two particular classes of copulas that proved to be useful in dependence modeling are the elliptical and Archimedean classes $[7,13]$. The four copulas (one of the elliptical copulas, Gaussian copula, and three of the Archimedean copulas, Gumbel copula, Clayton copula, and Frank copula) are employed as the candidates of the Bayesian copula selection criteria. Finally, the chosen ones are used for constructing the mixed copula model. Among the chosen copulas, Gaussian copulas and Frank copulas are able to capture symmetric dependence structures among random variables. Different from them, Gumbel copulas and Clayton copulas exhibit asymmetric dependence and Gumbel copulas are especially employed for describing upper tail dependence structures, while Clayton copulas are employed for that of the lower tail. And, then, the probability density function graphs and their contour plots are depicted as in Figures 1-4.

2.3. Modeling Method of Mixed Copula Models. There exist complex nonlinear correlations among failure modes; therefore, just one copula is not enough to characterize dependence structures among failure modes. With the aid of a weighted combination of the selected copula functions, a mixed copula is approximately constructed in order to describe the complex dependence structures among failure modes $[7,14]$; namely,

$$
C(\mathbf{u}, \mathbf{v} ; \mathbf{a}, \boldsymbol{\theta})=\sum_{i=1}^{N} a_{i} C_{i}\left(\mathbf{u}, \mathbf{v} ; \theta_{i}\right) \quad(N \leq 4),
$$

where $C_{i}(i=1,2, \ldots, N)$ are, respectively, denoted as one of the chosen copulas among the four copulas and $\mathbf{a}=$ $\left(a_{1}, a_{2}, \ldots, a_{N}\right)$ is the weighting coefficient vector along with the inner parameter vector $\boldsymbol{\theta}=\left(\theta_{1}, \theta_{2}, \ldots, \theta_{N}\right)$ of the corresponding copulas, and $\mathbf{u}, \boldsymbol{v}$ are variables of the chosen copula functions, and what is more, $a_{1}+a_{2}+\cdots+a_{N}=1$.

The copula function and Monte Carlo Simulation (MCS) method are contributed to the mixed copula model analysis. The specific steps can be stated as follows.

2.3.1. Monte Carlo Sampling (MCS). According to the distribution types of random variables for the performance functions of failure modes, the samples for the random variables can be obtained with MCS method. After substituting the samples into the corresponding performance functions, we can get the random sequence $\left\{g_{j}\right\}_{i}, i=$ $1,2, \ldots, n, j=1,2, \ldots, N$, for each of the performance functions and obtain the sequences $\left\{F_{j}\right\}_{i}=F\left(\left\{g_{j}\right\}_{i}\right), i=$ $1,2, \ldots, n, j=1,2, \ldots, N$, of the corresponding empirical 

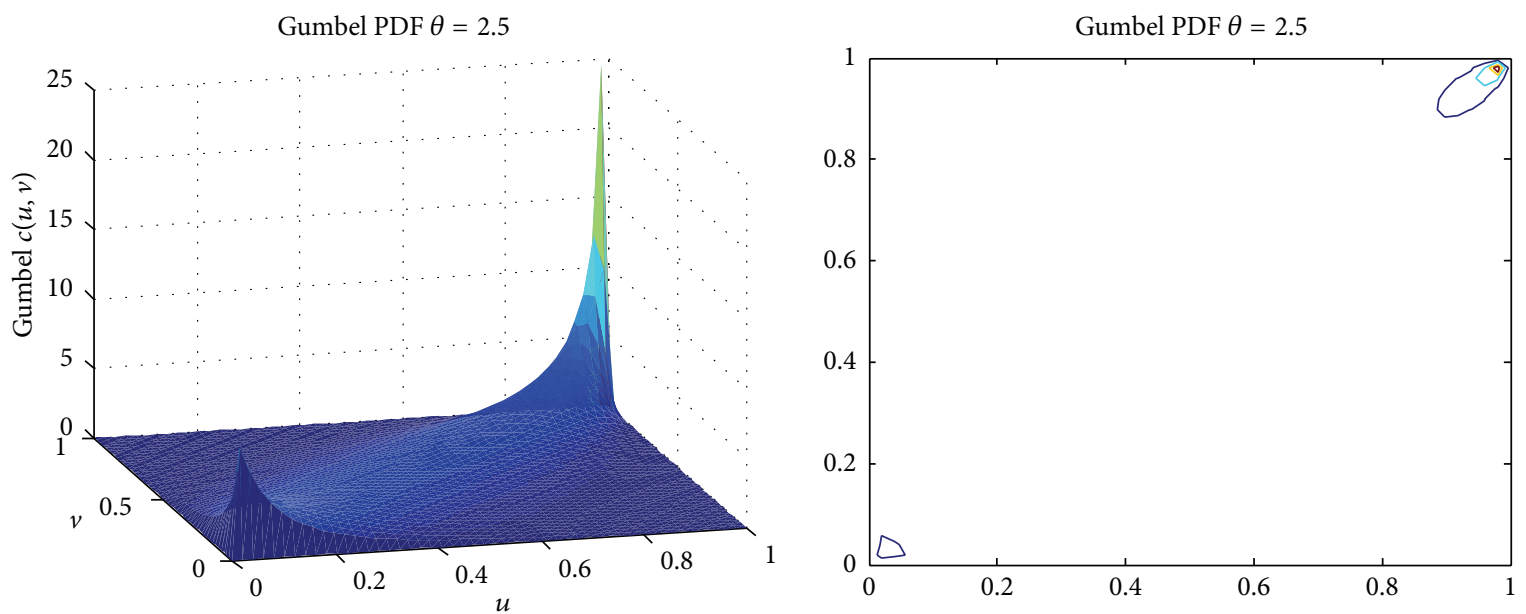

FIGURE 2: PDF and contour plots of Gumbel copula function.
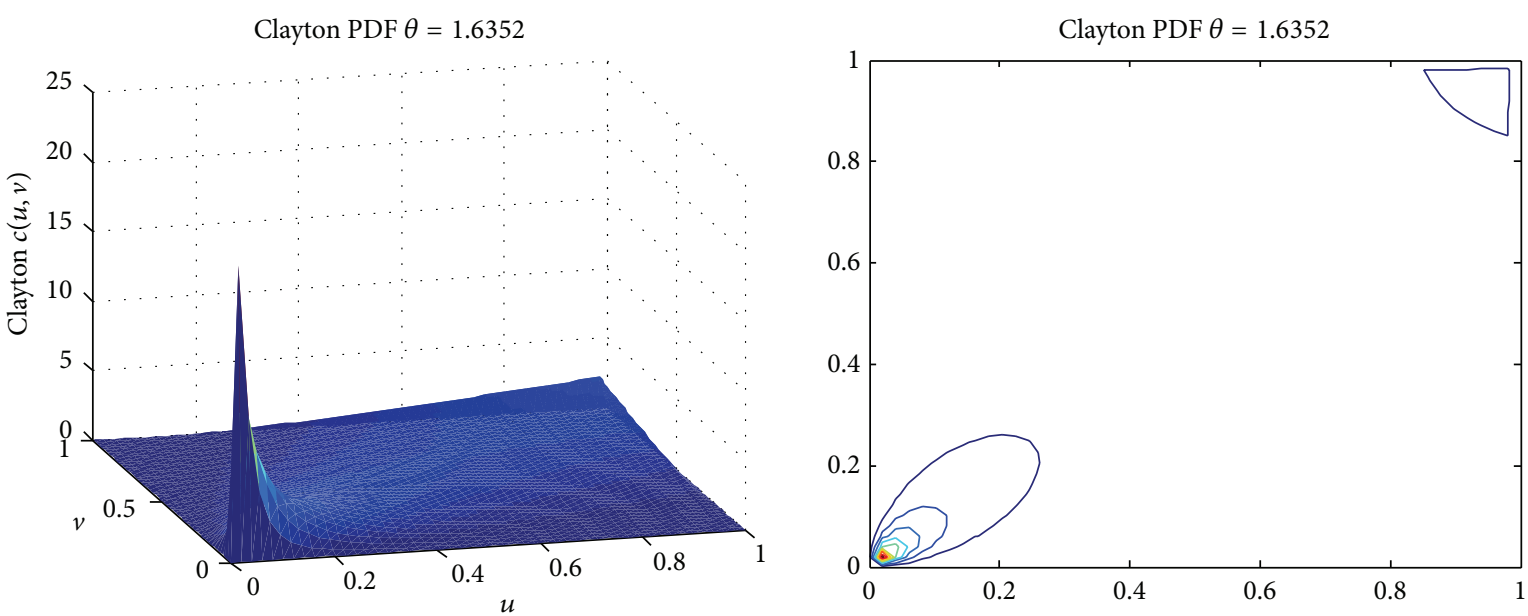

FIgURE 3: PDF and contour plots of Clayton copula function.
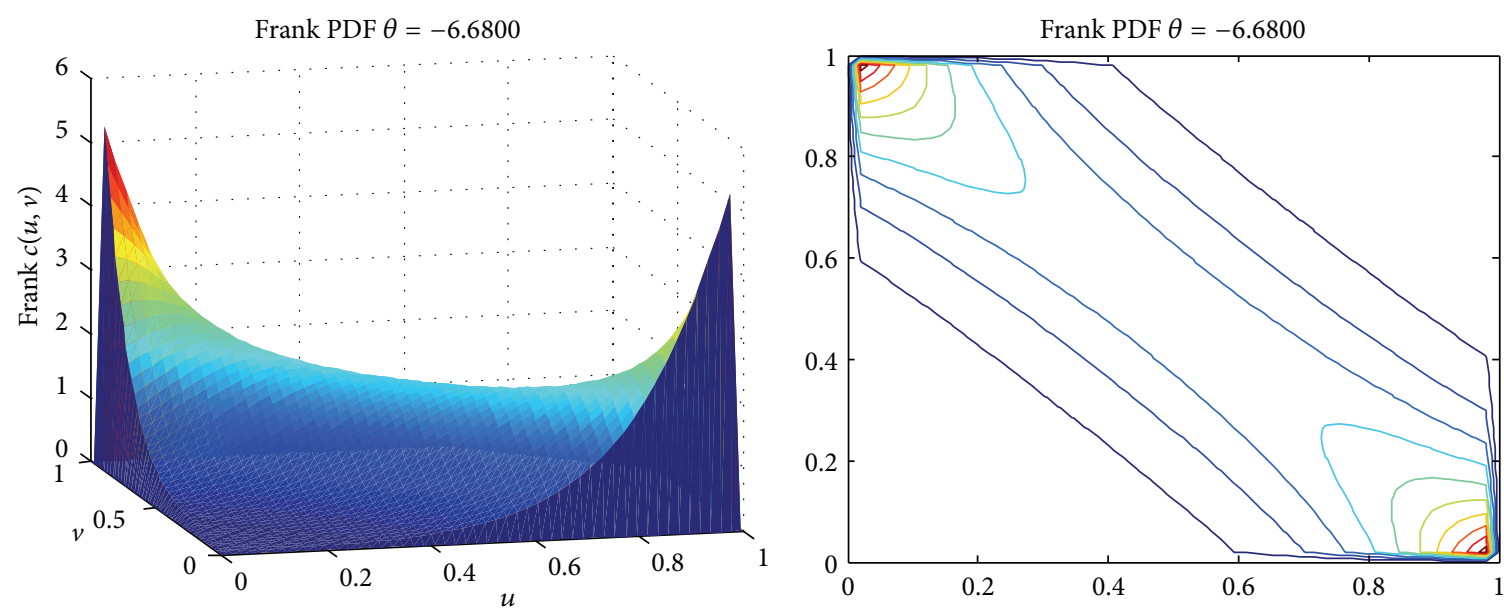

FIGURE 4: PDF and contour plots of Frank copula function. 
TABLE 1: Basic cases of the parameters of copula functions.

\begin{tabular}{lccc}
\hline Copula & $\tau=g(\theta)$ & $\tau \in \Omega$ & $\theta \in \Omega$ \\
\hline Gaussian & $\frac{2}{\pi} \arcsin \theta$ & {$[-1,1]$} & {$[-1,1]$} \\
Gumbel & $1-\theta^{-1}$ & {$[0,1]$} & $(0,1]$ \\
Clayton & $1-\frac{2}{2+\theta}$ & {$[1,+\infty)$} & $(0,+\infty)$ \\
Frank & $1-\frac{4}{\theta}\left(1-\frac{1}{\theta} \int_{0}^{\theta} \frac{t}{e^{t}-1} d t\right)$ & {$[-1,0) \cup(0,1]$} & $(-\infty, 0) \cup(0,+\infty)$ \\
\hline
\end{tabular}

distribution function by mean of Matlab software, where $g$ is the performance function and $F$ is the empirical distribution function.

2.3.2. Copula Function Selection. With the scatter plot of samples, we can judge approximate distribution features of the samples. Furthermore, it is extremely vital to determine which classes of copula functions to choose as the candidate copulas with Bayesian copula selection criteria. In this paper, the copula classes we chose are Gaussian copulas, Gumbel copulas, and Clayton copulas as well as Frank copulas, whose parameter domain along with the definition and the domain of Kendall's $\tau$ are listed in Table 1. The next is to build the mixed copula function through the selected copulas and (13).

2.3.3. Parameter Estimation for the Mixed Copula. Applying the least residual error quadratic sum method OLS, one of the curve fitting criteria, we can obtain parameters of the mixed copula function. And the formula of the OLS is represented as follows:

$$
F_{\mathrm{OLS}}=\sqrt{\frac{1}{n} \sum_{i=1}^{n}\left(F\left(\left(F_{1}\right)_{i},\left(F_{2}\right)_{i}, \ldots,\left(F_{N}\right)_{i}\right)-C\left(\left(F_{1}\right)_{i},\left(F_{2}\right)_{i}, \ldots,\left(F_{N}\right)_{i}\right)\right)^{2}},
$$

where $F(\cdot)$ is the joint empirical distribution function of the empirical distribution function sequences for each performance function and $C\left(\left(F_{1}\right)_{i},\left(F_{2}\right)_{i}, \ldots,\left(F_{N}\right)_{i}\right)$ is the mixed copula function value. The unknown parameter value for the mixed copula function can be obtained by the optimization computation with the rule of OLS, while the optimized parameters must be determined to make sure that the value of $F_{\mathrm{OLS}}$ is the minimum.

\subsubsection{Goodness-of-Fit Estimation for the Mixed Copula.} Through MCS method, the values of joint empirical distribution functions and the corresponding mixed copula function are obtained, and, then, the scatter plot between them is drawn. Finally, the goodness of fit for the mixed copula can be determined through the scatter plot.

\section{Mixed Copula Model Expressions about Joint Failure Probability of Structural System}

3.1. Mixed Copula Model Expression about Joint Failure Probability of Two-Component Series System. For the twocomponent series system which is shown in Figure 5, suppose that the performance function of the component failure mode is

$$
g_{i}(\mathbf{X})=g_{i}\left(\mathrm{X}_{1}, X_{2}, \ldots, X_{n}\right), \quad i=1,2 .
$$

With (5), as a check, the probability that both failure modes occur is denoted as

$$
\begin{aligned}
& P\left(g_{1}(\mathbf{X}) \leq 0, g_{2}(\mathbf{X}) \leq 0\right) \\
& =P\left(F_{g_{1}}\left(g_{1}(\mathbf{X})\right) \leq F_{g_{1}}(0), F_{g_{2}}\left(g_{2}(\mathbf{X})\right) \leq F_{g_{2}}(0)\right) \\
& =P\left(U_{1} \leq F_{g_{1}}(0), U_{2} \leq F_{g_{2}}(0)\right) \\
& =C\left(F_{g_{1}}(0), F_{g_{2}}(0)\right)=C\left(p_{f_{g_{1}}}, p_{f_{g_{2}}}\right) . \\
& F_{\mathbf{g}}(0,0)=C\left(F_{g_{1}}(0), F_{g_{2}}(0)\right) \text { is stated in the fundamental } \\
& \text { theorem of Sklar. Therefore, the failure probability of two- } \\
& \text { component series system (at least one failure mode of the two } \\
& \text { components occurred }) \text { can be solved as } \\
& \qquad p_{f}=P\left(g_{1}(\mathbf{X}) \leq 0 \cup g_{2}(\mathbf{X}) \leq 0\right) \\
& =P\left(g_{1}(\mathbf{X}) \leq 0\right)+P\left(g_{2}(\mathbf{X}) \leq 0\right) \\
& \quad-P\left(g_{1}(\mathbf{X}) \leq 0, g_{2}(\mathbf{X}) \leq 0\right) \\
& =p_{f_{g_{1}}}+p_{f_{g_{2}}}-C\left(p_{f_{g_{1}}}, p_{f_{g_{2}}}\right),
\end{aligned}
$$
theorem of Sklar. Therefore, the failure probability of twocomponent series system (at least one failure mode of the two

where $p_{f_{g_{1}}}, p_{f_{g_{2}}}$, respectively, denote the failure probability of the two failure modes and $C(\cdot)$ is two-component series system copula function.

3.2. Mixed Copula Model Expression about Joint Failure Probability of Two-Component Parallel System. For the twocomponent parallel system which is shown in Figure 6, 


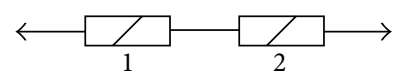

Figure 5: Two-component series system.

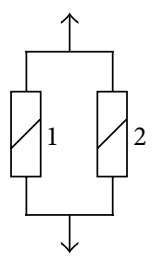

FIgURE 6: Two-component parallel system.

suppose that the performance function of the component failure mode is

$$
g_{i}(\mathbf{X})=g_{i}\left(X_{1}, X_{2}, \ldots, X_{n}\right), \quad i=1,2 .
$$

With (5) and (16), the failure probability of twocomponent parallel system (two failure modes of the two components meantime occurred) can be obtained:

$$
p_{f}=P\left(g_{1}(\mathbf{X}) \leq 0 \cap g_{2}(\mathbf{X}) \leq 0\right)=C\left(p_{f_{g_{1}}}, p_{f_{g_{2}}}\right),
$$

where $p_{f_{g_{1}}}, p_{f_{g_{2}}}$, respectively, denote the failure probability of the two failure modes and $C(\cdot)$ is two-component parallel system copula function.

3.3. Mixed Copula Model Expression about Joint Failure Probability of Multiple-Component Series System. For the multiplecomponent series system which is shown in Figure 7, suppose that the performance function of the component failure mode is

$$
g_{i}(\mathbf{X})=g_{i}\left(X_{1}, X_{2}, \ldots, X_{n}\right), \quad i=1,2, \ldots, N
$$

With (5), as a check, the probability that all the failure modes meantime occur is denoted as

$$
\begin{aligned}
P & \left(g_{1}(\mathbf{X}) \leq 0, g_{2}(\mathbf{X}) \leq 0, \ldots, g_{N}(\mathbf{X}) \leq 0\right) \\
& =P\left(F_{g_{1}}\left(g_{1}(\mathbf{X})\right) \leq F_{g_{1}}(0), F_{g_{2}}\left(g_{2}(\mathbf{X})\right)\right. \\
& \left.\leq F_{g_{2}}(0), \ldots, F_{g_{N}}\left(g_{N}(\mathbf{X})\right) \leq F_{g_{N}}(0)\right)=P\left(U_{1}\right. \\
& \left.\leq F_{g_{1}}(0), U_{2} \leq F_{g_{2}}(0), \ldots, U_{N} \leq F_{g_{N}}(0)\right) \\
& =C\left(F_{g_{1}}(0), F_{g_{2}}(0), \ldots, F_{g_{N}}(0)\right) \\
& =C\left(p_{f_{g_{1}}}, p_{f_{g_{2}}}, \ldots, p_{f_{g_{N}}}\right) .
\end{aligned}
$$

$F_{\mathbf{g}}(0,0, \ldots, 0)=C\left(F_{g_{1}}(0), F_{g_{2}}(0), \ldots, F_{g_{N}}(0)\right)$ is stated in the fundamental theorem of Sklar. Therefore, the failure probability of multiple-component series system (at least one

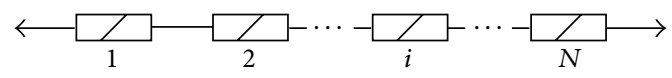

FIGURE 7: Multiple-component series system.

failure mode of the multiple components occurred) can be solved as

$$
\begin{aligned}
p_{f} & =P\left(g_{1}(\mathbf{X}) \leq 0 \cup g_{2}(\mathbf{X}) \leq 0 \cup \cdots \cup g_{N}(\mathbf{X}) \leq 0\right) \\
& =\sum_{i=1}^{N} P\left(g_{i}(\mathbf{X}) \leq 0\right)-\sum_{i_{1}<i_{2}} P\left(g_{i_{1}}(\mathbf{X}) \leq 0, g_{i_{2}}(\mathbf{X})\right. \\
& \leq 0)+\cdots+(-1)^{n+1} \sum_{i_{1}<i_{2}<\cdots<i_{n}} P\left(g_{i_{1}}(\mathbf{X}) \leq 0, g_{i_{2}}(\mathbf{X})\right. \\
& \left.\leq 0, \ldots, g_{i_{n}}(\mathbf{X}) \leq 0\right)+\cdots+(-1)^{N+1} P\left(g_{1}(\mathbf{X}) \leq 0,\right. \\
& \left.g_{2}(\mathbf{X}) \leq 0, \ldots, g_{N}(\mathbf{X}) \leq 0\right)=\sum_{i=1}^{n} p_{g_{g_{i}}} \\
& -\sum_{i_{1}<i_{2}} C\left(p_{f_{g_{i_{1}}}}, p_{f_{g_{i_{2}}}}\right)+\cdots+(-1)^{n+1} \\
& \cdot \sum_{i_{1}<i_{2}<\cdots<i_{n}} C\left(p_{f_{g_{i_{1}}}}, p_{f_{g_{i_{2}}}}, \ldots, p_{f_{g_{i_{n}}}}\right)+\cdots \\
& +(-1)^{N+1} C\left(p_{f_{g_{1}}}, p_{f_{g_{2}}}, \ldots, p_{f_{g_{N}}}\right),
\end{aligned}
$$

where $p_{f_{g_{1}}}, p_{f_{g_{2}}}, \ldots, p_{f_{g_{N}}}$, respectively, denote the failure probability of the multiple failure modes and $C(\cdot)$ is multiplecomponent series system copula function.

3.4. Mixed Copula Model Expression about Joint Failure Probability of Multiple-Component Parallel System. For the multiple-component parallel system which is shown in Figure 8 , suppose that the performance function of the component failure mode is

$$
g_{i}(\mathbf{X})=g_{i}\left(X_{1}, X_{2}, \ldots, X_{n}\right), \quad i=1,2, \ldots, N .
$$

With (5), the failure probability of two-component parallel system (all the failure modes of all the components meantime occurred) can be obtained:

$$
\begin{aligned}
p_{f} & =P\left(g_{1}(\mathbf{X}) \leq 0, g_{2}(\mathbf{X}) \leq 0, \ldots, g_{N}(\mathbf{X}) \leq 0\right) \\
& =P\left(F_{g_{1}}\left(g_{1}(\mathbf{X})\right) \leq F_{g_{1}}(0), F_{g_{2}}\left(g_{2}(\mathbf{X})\right)\right. \\
& \left.\leq F_{g_{2}}(0), \ldots, F_{g_{N}}\left(g_{N}(\mathbf{X})\right) \leq F_{g_{N}}(0)\right)=P\left(U_{1}\right. \\
& \left.\leq F_{g_{1}}(0), U_{2} \leq F_{g_{2}}(0), \ldots, U_{N} \leq F_{g_{N}}(0)\right) \\
& =C\left(F_{g_{1}}(0), F_{g_{2}}(0), \ldots, F_{g_{N}}(0)\right) \\
& =C\left(p_{f_{g_{1}}}, p_{f_{g_{2}}}, \ldots, p_{g_{g_{N}}}\right),
\end{aligned}
$$

where $p_{f_{g_{1}}}, p_{f_{g_{2}}}, \ldots, p_{f_{g_{N}}}$, respectively, denote the failure probability of the $N$ failure modes and $C(\cdot)$ is multiplecomponent parallel system copula function. 


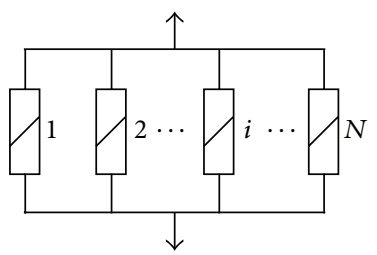

FIGURE 8: Multiple-component parallel system.

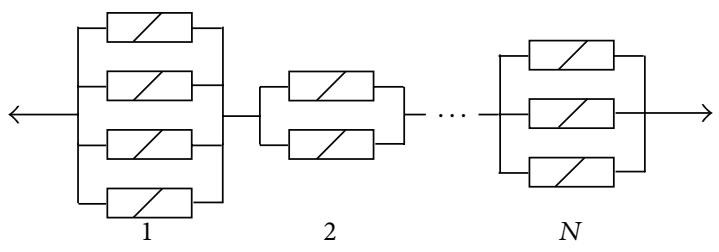

Figure 9: Series-parallel system.

3.5. Mixed Copula Model Expression about Joint Failure Probability of Series-Parallel System. For series-parallel system shown in Figure 9, in this paper, only the correlation between internal components of each subparallel system is considered, while the correlation between subparallel systems is not considered and considered to be mutually independent. Therefore, with (24), all the copula models of all the subparallel systems' failure probability can be obtained, and then, with (25), the failure probability of the series-parallel system can be solved:

$$
\begin{aligned}
p_{f_{\text {s-p }}}= & P\left(\bigcup_{i=1}^{N} F_{i}\right)=P\left(\bigcup_{i=1}^{N} \bigcap_{j}^{N_{i}} F_{i j}\right) \\
= & \sum_{i=1}^{N} P\left(F_{i}\right)-\sum_{i_{1}<i_{2}}\left\{P\left(F_{i_{1}}\right) P\left(F_{i_{2}}\right)\right\}+\cdots \\
& +(-1)^{n+1} \sum_{i_{1}<i_{2}<\cdots<i_{n}}\left\{\prod_{k=i_{1}}^{i_{n}} P\left(F_{k}\right)\right\}+\cdots \\
& +(-1)^{N+1} \prod_{i=1}^{N} P\left(F_{i}\right),
\end{aligned}
$$

where $N$ is the total number of the subparallel systems; $N_{i}$ is the total number of the components in the $i$ th subparallel system; $F_{i}=\bigcap_{j=1}^{N_{i}} F_{i j}$ is the failure probability of the $i$ th subparallel system; $F_{i j}=\left(g_{i j}(\mathbf{X}) \leq 0\right)$ is the failure probability of the $j$ th component in the $i$ th subparallel system; $P\left(F_{i}\right), i=$ $1,2, \ldots, N$, can be solved with $(24)$, which are the failure probability of the subparallel system considering correlation between internal components of each subparallel system.

3.6. Mixed Copula Model Expression about Joint Failure Probability of Parallel-Series System. For parallel-series system shown in Figure 10, in this paper, only the correlation between internal components of each subseries system is considered, while the correlation between subseries systems is not considered and considered to be mutually independent.

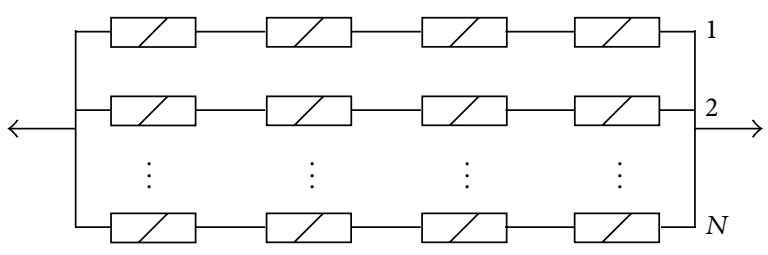

Figure 10: Parallel-series system.

Therefore, with (22), all the copula models of all the subseries systems' failure probability can be obtained, and then, with (26), the failure probability of the parallel-series system can be solved as

$$
p_{f_{\mathrm{p}-\mathrm{s}}}=P\left(\bigcap_{i=1}^{N} F_{i}\right)=\prod_{i=1}^{N} P\left(F_{i}\right)=\prod_{i=1}^{N} P\left(\bigcup_{j=1}^{N_{i}} F_{i j}\right),
$$

where $N$ is the total number of the subseries systems; $N_{i}$ is the total number of the components in the $i$ th subseries system; $F_{i}=\bigcap_{j=1}^{N_{i}} F_{i j}$ is the failure probability of the $i$ th subseries system; $F_{i j}=\left(g_{i j}(\mathbf{X}) \leq 0\right)$ is the failure probability of the $j$ th component in the $i$ th subseries system; $P\left(F_{i}\right), i=1,2, \ldots, N$, can be solved with (22), which are the failure probability of the subseries system considering correlation between internal components of each subseries system.

In this paper, firstly with First-Order Reliability Method (FORM), the structural reliability index and the corresponding failure probability of each failure mode can be solved, and then with the constructed mix copula functions, such as (17), (19), (22), (24), (25), and (26), the failure probability of structural system considering correlation between failure modes can be obtained.

\section{Numerical Example: System Reliability Analysis of Simply Supported Cored Slab Bridge}

For the simply supported cored slab bridge shown in Figure 11 , the total span is $13 \mathrm{~m}$, the computed span is $12.6 \mathrm{~m}$, the clear width of bridge deck is $7 \mathrm{~m}$, the width of footway on both sides of bridge deck is $1 \mathrm{~m}$, and the whole bridge is composed of nine concrete cored slabs [15]. The design reference period of this bridge is 100 years. And this bridge has been served for 32 years. At the 32 nd year, the resistance of each girder follows normal distribution; the distribution parameters are, respectively, $796.04 \mathrm{kN} \cdot \mathrm{m}$ (mean value) and $91.783 \mathrm{kN} \cdot \mathrm{m}$ (standard deviation).

Based on Figure 11 and reference [16], the failure criterion of bridge system is as follows: if any two adjacent girders both failed, then the whole bridge system failed. According to the failure criterion, the bridge system is a series-parallel system, which is shown in Figure 12.

The performance function of each girder's failure mode is

$$
\begin{array}{r}
Z_{i}=g\left(X_{i 1}, X_{i 2}, X_{i 3}\right)=g\left(R_{i}, G_{i}, Q_{i}\right)=R_{i}-G_{i}-Q_{i}, \\
i=1,2, \ldots, 9,
\end{array}
$$


TABLE 2: Distribution parameters of beams' dead load effects in 32nd year.

\begin{tabular}{|c|c|c|c|c|c|c|c|c|c|}
\hline Girder number & 1 & 2 & 3 & 4 & 5 & 6 & 7 & 8 & 9 \\
\hline Mean value $/ \mathrm{kN} \cdot \mathrm{m}$ & 212.86 & 221.79 & 223.91 & 230.74 & 260.69 & 230.74 & 223.91 & 221.79 & 212.86 \\
\hline Standard deviation $/ \mathrm{kN} \cdot \mathrm{m}$ & 9.68 & 9.68 & 9.68 & 9.68 & 9.68 & 9.68 & 9.68 & 9.68 & 9.68 \\
\hline
\end{tabular}

TABLE 3: Distribution parameters of beams' maximum live load effects in 32nd year.

\begin{tabular}{lccccccccc}
\hline Girder number & 1 & 2 & 3 & 4 & 5 & 6 & 7 & 8 & 9 \\
\hline Mean value/kN·m & 85.42 & 96.42 & 116.8 & 144.4 & 180.9 & 144.4 & 116.8 & 96.42 & 85.42 \\
Standard deviation $/ \mathrm{kN} \cdot \mathrm{m}$ & 12.71 & 14.35 & 17.38 & 21.49 & 26.92 & 21.49 & 17.38 & 14.35 & 12.71 \\
\hline
\end{tabular}

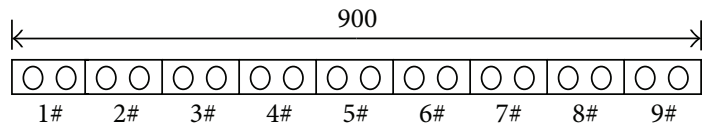

FIgURE 11: Cross section of bridge (unit: $\mathrm{cm}$ ).

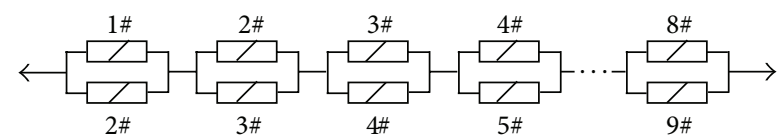

Figure 12: Series-parallel system.

where $R_{i}$ is the resistance of the $i$ th girder, $G_{i}$ is the dead load effect of the $i$ th girder, and $Q_{i}$ is the live load effect of the $i$ th girder. At the 32nd year, the distribution parameters about dead load effects are listed in Table 2, where the standard deviation is not changed, because, for load effect, the variation of variables is very small. And the distribution parameters about vehicle load effects are listed in Table 3, which occur due to symmetrical variable load and is not applicated to the unsymmetrical variable load.

Based on the reliability analysis method of twocomponent parallel system considering the correlation between failures modes described in Section 3.2, the reliability analysis processes of bridge system are as follows.

Because the bridge system is symmetrical, the subparallel systems (1\#-2\#, 2\#-3\#, 3\#-4\#, and 4\#-5\#) are used to analyze the reliability and failure probability of the bridge system.

Based on Tables 2 and 3 and (25), the scatter plot between two random sampling sequences of the corresponding limit state functions for the two failure modes of each subparallel system (1\#-2\#, 2\#-3\#, 3\#-4\#, and 4\#-5\#) can be obtained. Then, according to the characteristics of the obtained scatter plots, the candidate copula functions, which can approximately describe distribution features of the samples, can be selected, and then with Bayesian selection criteria described in Section 2.2, the suitable copula functions, from the selected candidate copula functions, can be obtained which can be used to build the mixed copula model. The parameters of the built mixed copula function for each subparallel system are listed in Table 4. Finally, the mixed copula modes, for
TABle 4: Parameters of each of the mixed copulas for four subsystems.

\begin{tabular}{ccccccc}
\hline & $a$ & $b$ & $1-a-b$ & $\rho$ & $\alpha$ & $\theta$ \\
\hline 1\#-2\# & 0.7438 & 0.1958 & 0.0604 & -0.1868 & 2.4872 & 4.4278 \\
2\#-3\# & 0.8521 & 0.1479 & 0 & 0.1014 & 2.4207 & 4.4078 \\
3\#-4\# & 0.9381 & 0.0619 & 0 & 0.1502 & 2.4611 & 4.3984 \\
4\#-5\# & 0.7690 & 0.2310 & 0 & -0.3490 & 2.4814 & 4.4192 \\
\hline
\end{tabular}

each subparallel system, are built. Further, PDF plots and contour plots for the mixed copulas, the scatter plots between empirical distributions and mixed copula functions, and scatter plots for two limit state functions are presented, respectively, in Figures 13-16.

With FORM, the corresponding reliability indices to each girder's failure mode are, respectively,

$$
\begin{aligned}
& \beta_{1}=5.3429, \\
& \beta_{2}=5.1159, \\
& \beta_{3}=4.8484, \\
& \beta_{4}=4.4417, \\
& \beta_{5}=3.6869 .
\end{aligned}
$$

Then, with the equation $p_{f}=\Phi(-\beta)$, the corresponding failure probability to each girder's failure mode is, respectively,

$$
\begin{aligned}
& p_{f_{g_{1}}}=4.57 \times 10^{-8}, \\
& p_{f_{g_{2}}}=1.56 \times 10^{-7}, \\
& p_{f_{g_{3}}}=6.22 \times 10^{-7}, \\
& p_{f_{g_{4}}}=4.46 \times 10^{-6}, \\
& p_{f_{g_{5}}}=1.14 \times 10^{-4} .
\end{aligned}
$$




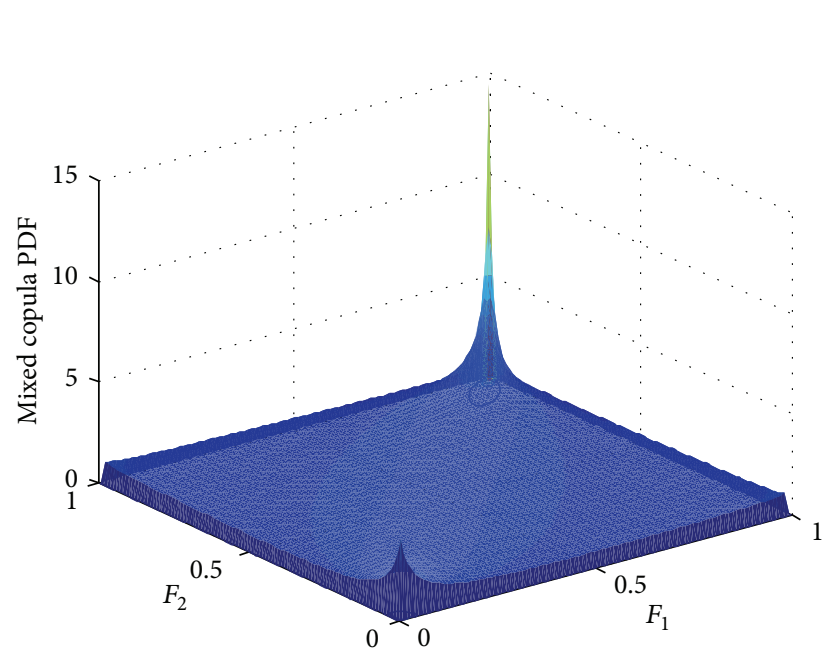

(a) PDF plot for the mixed copula

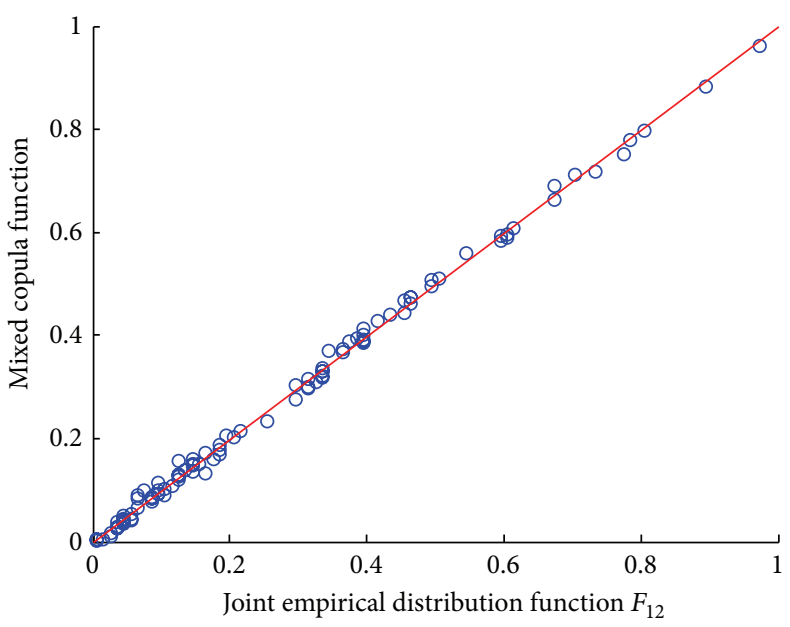

(c) Empirical distribution versus mixed copula

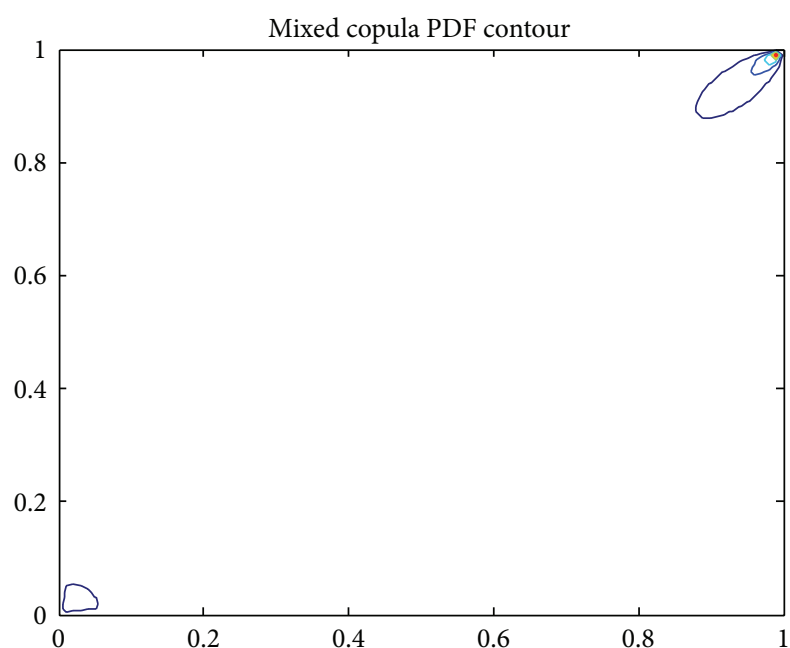

(b) Contour plot

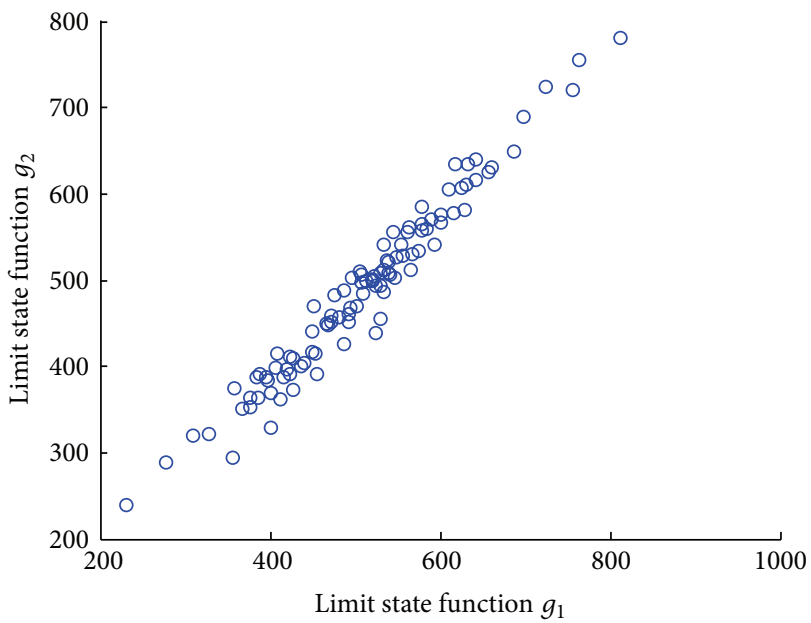

(d) Scatter plot for two limit state functions

FIGURE 13: The subsystem 1\#-2\#.

With (19) and Table 4, the following can be obtained:

$$
\begin{aligned}
& p_{f_{12}}=C\left(p_{f_{1}}, p_{f_{2}}\right)=8.61 \times 10^{-11}, \\
& p_{f_{23}}=C\left(p_{f_{2}}, p_{f_{3}}\right)=3.12 \times 10^{-10}, \\
& p_{f_{34}}=C\left(p_{f_{3}}, p_{f_{4}}\right)=1.32 \times 10^{-9}, \\
& p_{f_{45}}=C\left(p_{f_{4}}, p_{f_{5}}\right)=1.31 \times 10^{-7},
\end{aligned}
$$

where $p_{f_{12}}$ is the failure probability when girder $1 \#$ and girder $2 \#$ meantime failed, $p_{f_{23}}$ is the failure probability when girder 2\# and girder $3 \#$ meantime failed, $p_{f_{34}}$ is the failure probability when girder $3 \#$ and girder $4 \#$ meantime failed, and $p_{f_{45}}$ is the failure probability when girder $4 \#$ and girder $5 \#$ meantime failed.
Suppose that failure modes between subsystems are mutually independent, and then the failure probability of structural system is approximately

$$
\begin{aligned}
p_{f} & =(-1)^{2} \sum_{j=i+1, i=1}^{8} p_{f_{i j}}+(-1)^{3} \sum_{t=k+1, j=i+1, k>i, i=1}^{7} p_{f_{i j}} p_{f_{k t}} \\
& +\cdots+(-1)^{9}\left(p_{f_{12}} p_{f_{23}} p_{f_{34}} p_{f_{45}} p_{f_{56}} p_{f_{67}} p_{f_{78}} p_{f_{89}}\right) \\
& \approx \sum_{j=i+1, i=1}^{8} p_{f_{i j}}-\sum_{t=k+1, j=i+1, k>i, i=1}^{7} p_{f_{i j}} p_{f_{k t}}=2\left(p_{f_{12}}\right. \\
& \left.+p_{f_{23}}+p_{f_{34}}+p_{f_{45}}\right)-4\left(p_{f_{12}} p_{f_{23}}+p_{f_{12}} p_{f_{34}}\right. \\
& \left.+p_{f_{12}} p_{f_{45}}+p_{f_{23}} p_{f_{34}}+p_{f_{23}} p_{f_{45}}+p_{f_{34}} p_{f_{45}}\right)-p_{f_{12}}^{2} \\
& -p_{f_{23}}^{2}-p_{f_{34}}^{2}-p_{f_{45}}^{2}=2.65 \times 10^{-7},
\end{aligned}
$$




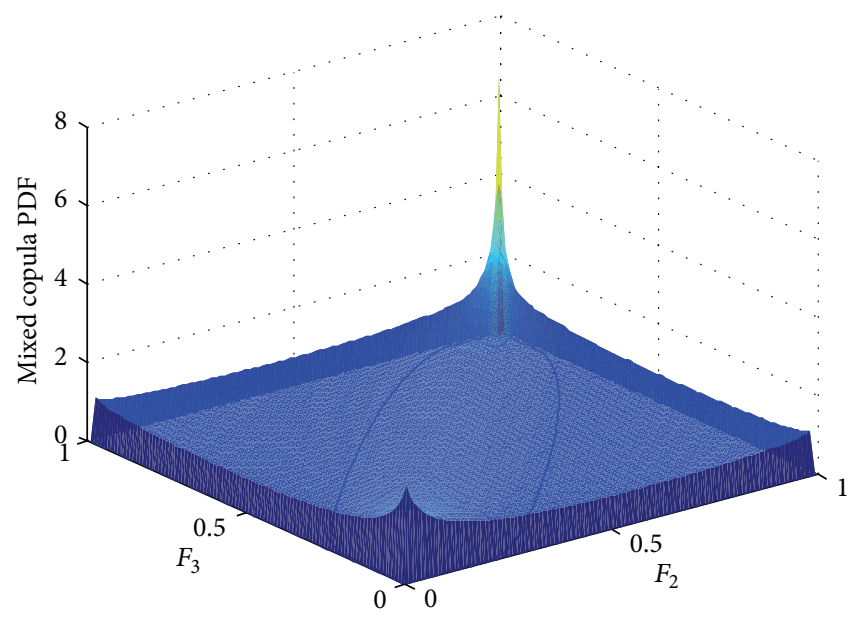

(a) PDF plot for the mixed copula

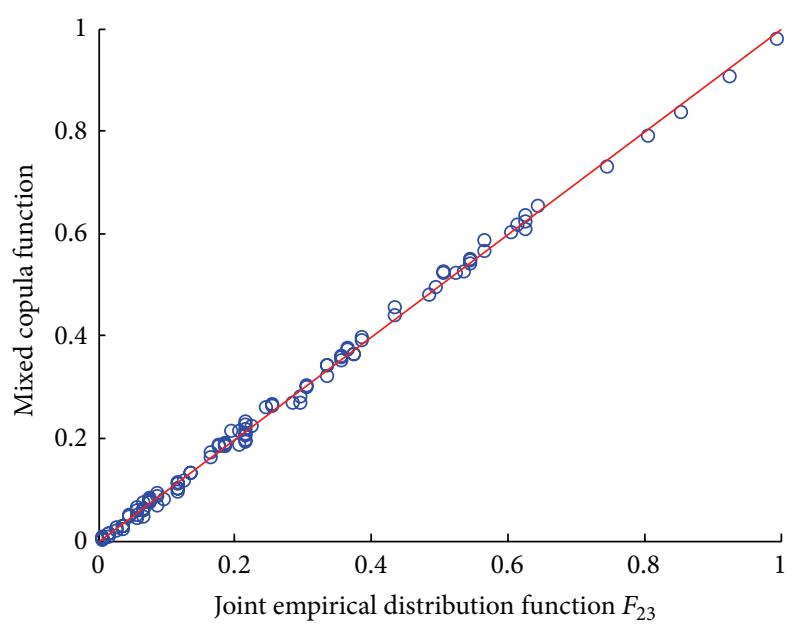

(c) Empirical distribution versus mixed copula

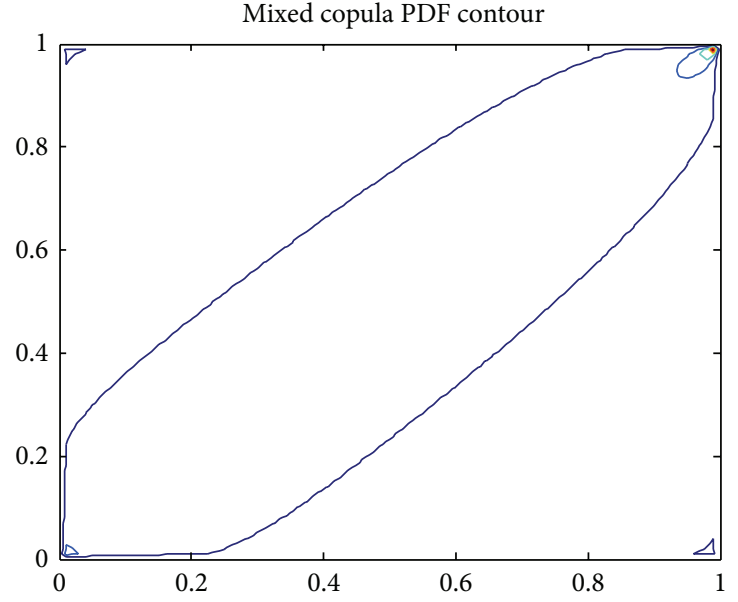

(b) Contour plot

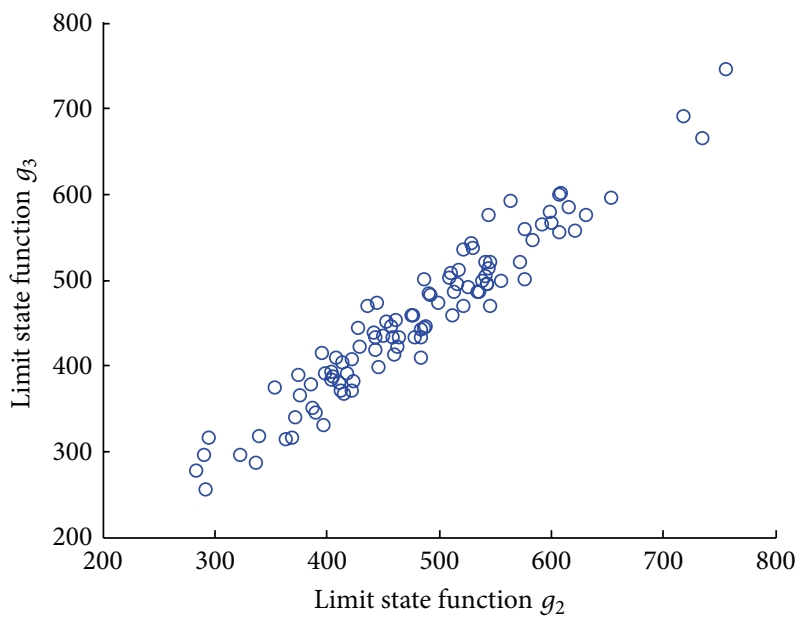

(d) Scatter plot for two limit state functions

FIgURE 14: The subsystem 2\#-3\#.

where, because the structural system is symmetric, the failure probability of the subsystems at symmetric positions is the same. Namely, $p_{f_{12}}=p_{f_{89}}, p_{f_{23}}=p_{f_{78}}, \ldots, p_{f_{45}}=$ $P_{f_{56}}{ }^{\circ}$ For each subparallel system, the failure probability without considering the correlation between the two components is

$$
\begin{aligned}
& h_{f_{12}}=p_{f_{1}} p_{f_{2}}=7.13 \times 10^{-15}, \\
& h_{f_{23}}=p_{f_{2}} p_{f_{3}}=9.70 \times 10^{-14}, \\
& h_{f_{34}}=p_{f_{3}} p_{f_{4}}=2.77 \times 10^{-12}, \\
& h_{f_{45}}=p_{f_{4}} p_{f_{5}}=5.08 \times 10^{-10} .
\end{aligned}
$$

Suppose that failure modes between subsystems are mutually independent, and the failure modes between the two components of each subsystem are also mutually independent; then, the failure probability of structural system [17] is approximately

$$
\begin{aligned}
p_{f} & =(-1)^{2} \sum_{j=i+1, i=1}^{8} h_{f_{i j}}+(-1)^{3} \sum_{t=k+1, j=i+1, k>i, i=1}^{7} h_{f_{i j}} h_{f_{k t}} \\
& +\cdots+(-1)^{9}\left(h_{f_{12}} h_{f_{23}} h_{f_{34}} h_{f_{45}} h_{f_{56}} h_{f_{67}} h_{f_{78}} h_{f_{89}}\right) \\
& \approx \sum_{j=i+1, i=1}^{8} h_{f_{i j}}-\sum_{t=k+1, j=i+1, k>i, i=1} h_{f_{i j}} h_{f_{k t}}=2\left(h_{f_{12}}\right. \\
& \left.+h_{f_{23}}+h_{f_{34}}+h_{f_{45}}\right)-4\left(h_{f_{12}} h_{f_{23}}+h_{f_{12}} h_{f_{34}}\right. \\
& \left.+h_{f_{12}} h_{f_{45}}+h_{f_{23}} h_{f_{34}}+h_{f_{23}} h_{f_{45}}+h_{f_{34}} h_{f_{45}}\right)-h_{f_{12}}^{2} \\
& -h_{f_{23}}^{2}-h_{f_{34}}^{2}-h_{f_{45}}^{2}=1.02 \times 10^{-9} .
\end{aligned}
$$

From the above solved system's failure probability shown in (31) and (33), it can be seen that the failure probability of the 


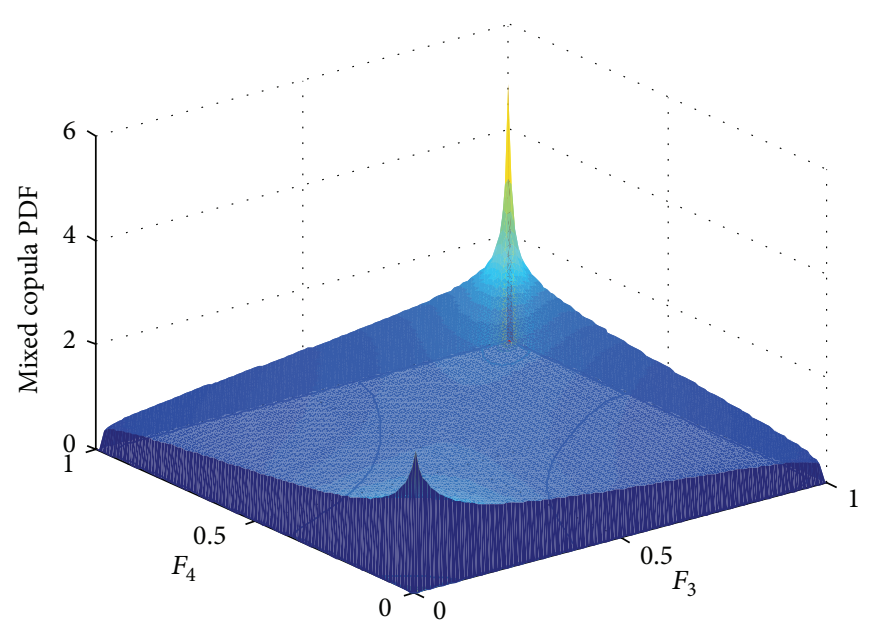

(a) PDF plot for the mixed copula

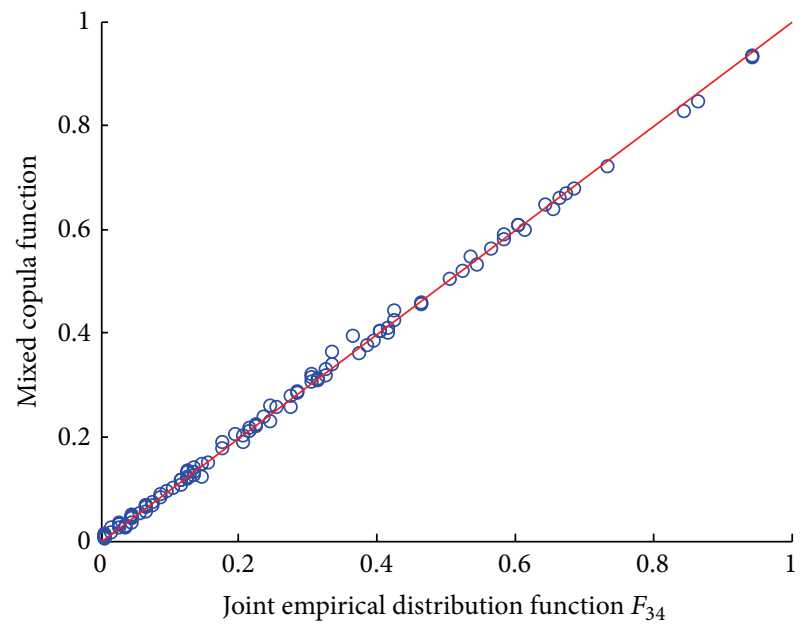

(c) Empirical distribution versus mixed copula

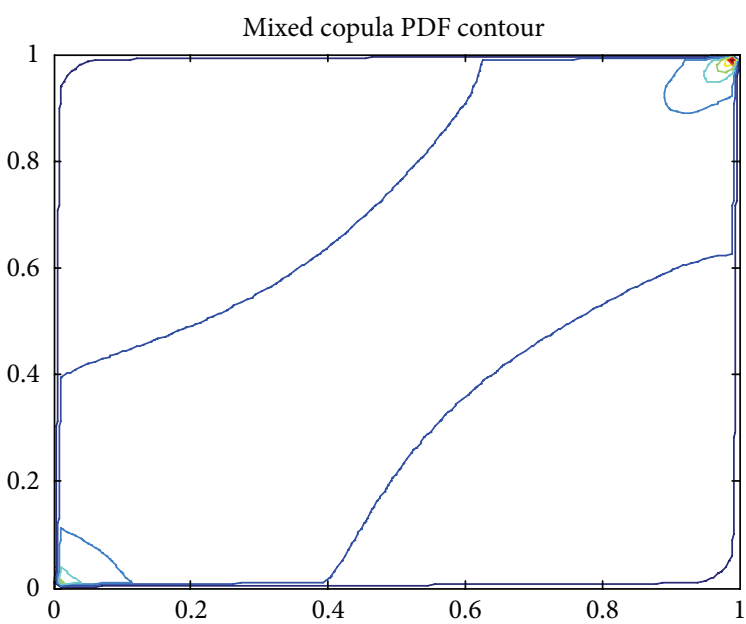

(b) Contour plot

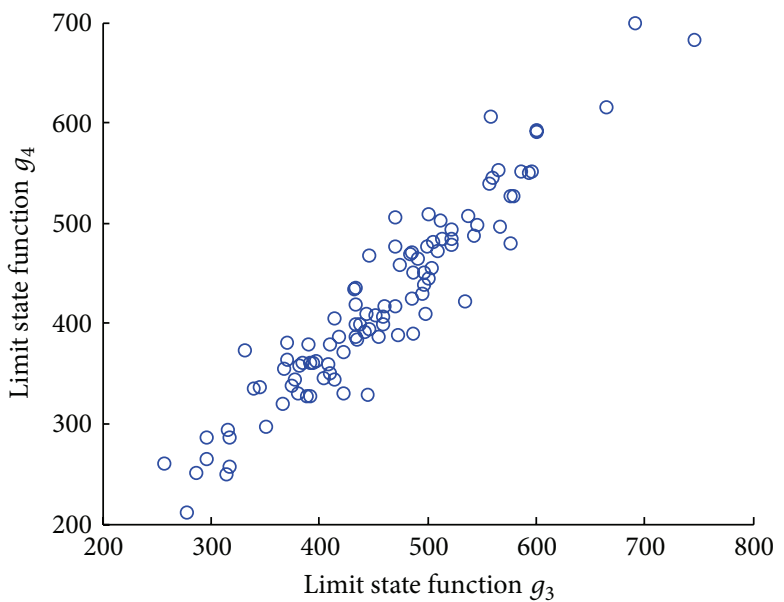

(d) Scatter plot for two limit state functions

Figure 15: The subsystem 3\#-4\#.

series-parallel system considering the correlation between two adjacent girders is larger than the failure probability without considering the correlation between two adjacent girders, which showed that the series-parallel system considering the correlation between two adjacent girders more easily failed. Further, it is illustrated that considering the correlation between two adjacent girders of each subparallel system is essential and applicable for solving the reliability of the series-parallel system.

\section{Conclusions}

For the two-component systems and multiple-component systems with multiple failure modes, this paper presents the mixed copula models for reliability analysis of series systems, parallel systems, series-parallel systems, and parallelseries systems. The mixed copula model is obtained with the chosen optimal copula functions with the Bayesian method. Through a numerical example, it is illustrated that the calculated failure probability when considering the correlation between failure modes is larger than that without considering the correlation between failure modes. It is verified that the solved failure probability is conservative without considering the correlation between failure modes.

This paper provided a new method for characterizing the correlation between failure modes and solving the reliability of the system considering correlation between failure modes.

\section{Competing Interests}

The authors declare that they have no competing interests.

\section{Acknowledgments}

This work was supported by the Fundamental Research Funds for the Central Universities (lzujbky-2015-300, lzujbky-2015-301). 


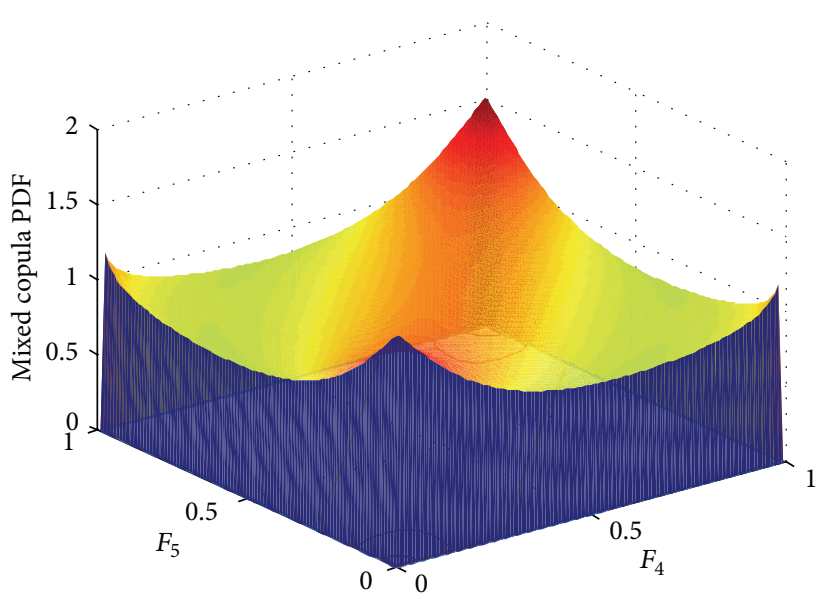

(a) PDF plot for the mixed copula

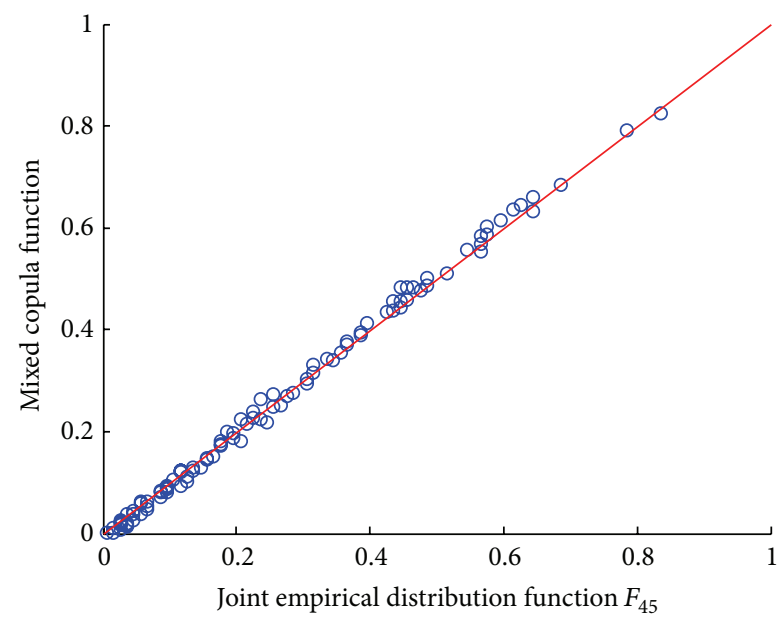

(c) Empirical distribution versus mixed copula

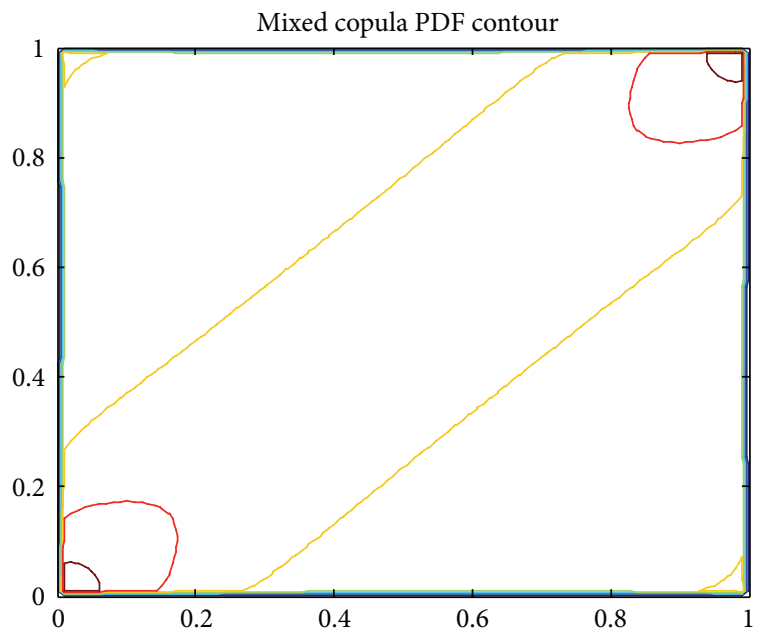

(b) Contour plot

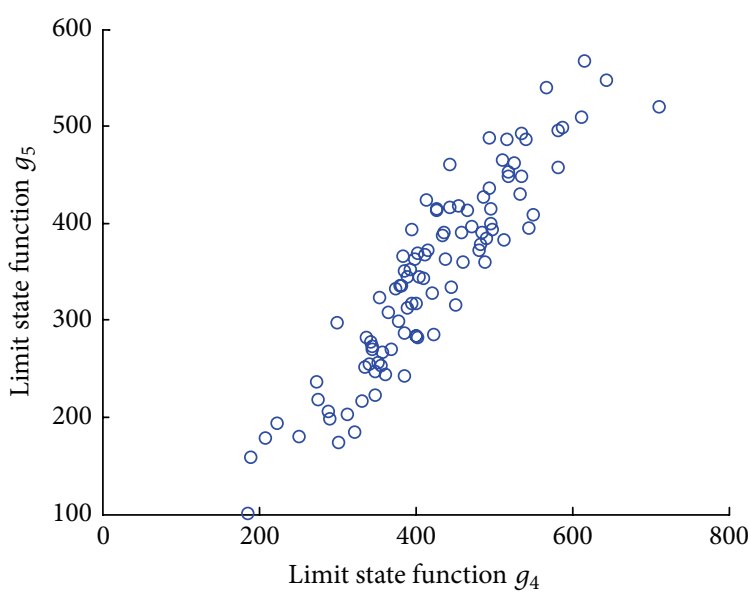

(d) Scatter plot for two limit state functions

FIgURE 16: The subsystem 4\#-5\#.

\section{References}

[1] S. Eryilmaz, "Multivariate copula based dynamic reliability modeling with application to weighted-k-out-of-n systems of dependent components," Structural Safety, vol. 51, pp. 23-28, 2014.

[2] A. S. Balu and B. N. Rao, "Multicut-high dimensional model representation for structural reliability bounds estimation under mixed uncertainties," Computer-Aided Civil and Infrastructure Engineering, vol. 27, no. 6, pp. 419-438, 2012.

[3] L. Dueñas-Osorio and J. Rojo, "Reliability assessment of lifeline systems with radial topology," Computer-Aided Civil and Infrastructure Engineering, vol. 26, no. 2, pp. 111-128, 2011.

[4] D. V. Val, M. G. Stewart, and R. E. Melchers, "Life-cycle performance of RC bridges: probabilistic approach," ComputerAided Civil and Infrastructure Engineering, vol. 15, no. 1, pp. 1425, 2000.

[5] G. Li and G.-D. Cheng, "Correlation between structural failure modes and calculation of system reliability under hazard loads," Engineering Mechanics, vol. 18, no. 3, pp. 1-9, 2001.

[6] D.-Q. Li and S.-B. Wu, "System reliability analysis on main girder of plane gates considering multiple correlated failure modes," Journal of Hydraulic Engineering, vol. 40, no. 7, pp. 870877, 2009 (Chinese).

[7] Y. Liu and D. Lu, "Reliability analysis of two-dimensional series portal-framed bridge system based on mixed copula functions," Key Engineering Materials, vol. 574, pp. 95-105, 2014.

[8] W. Q. Han, J. Y. Zhou, and K. Z. Sun, "Copulas in the mechanical components reliability analysis," in Reliability Technology Academic Exchange Meeting and the 4th National Machinery Industry Reliability Engineering Branch of the Second Plebary Committee Conference Proceedings, Xiangtan, China, 2010 (Chinese).

[9] Y. Y. Hou and S. B. Song, "Research of the joint distribution for flood peak and volume based on copula function," Yellow River, vol. 30, no. 11, pp. 39-44, 2010 (Chinese).

[10] D. Huard, G. Évin, and A.-C. Favre, "Bayesian copula selection," Computational Statistics \& Data Analysis, vol. 51, no. 2, pp. 809822, 2006.

[11] N. Fan, X. L. He, and Q. Zhao, "Optimal copula function selection based on Bayesian methodology," Chinese Journal of Engineering Mathematics, vol. 29, no. 4, pp. 516-522, 2012 (Chinese). 
[12] A. Sklar, "Fonctions de répartition à $\mathrm{n}$ dimensions et leurs marges," Publications de l'Institut de Statistique de l'Université de Paris, vol. 8, pp. 229-231, 1959.

[13] R. B. Nelsen, An Introduction to Copulas, Springer, New York, NY, USA, 2006.

[14] L. Hu, Essays in Econometrics with Applications in Macroeconomic and Financial Modelling, Yale University, New Haven, Conn, USA, 2002.

[15] X. P. Fan, Reliability Evaluation of Concrete Continuous Beam Bridge Based on Real-Time Monitoring Information, School of Civil Engineering, Harbin Institute of Technology, Harbin, China, 2010.

[16] E. S. Gharaibeh, Reliability and Redundancy of Structural Systems with Application to Highway Bridges, University of Colorado, Denver, Colo, USA, 1999.

[17] R. E. Melchers, Structural Reliability, Analysis and Prediction, Ellis Horwood, Chichester, UK, 1987. 


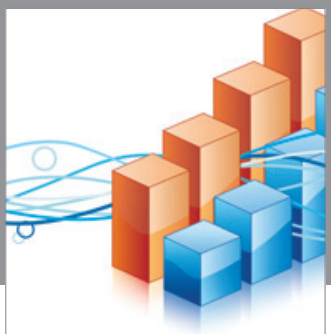

Advances in

Operations Research

vatem alat4

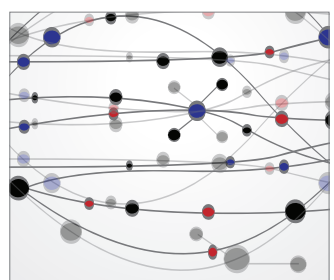

\section{The Scientific} World Journal
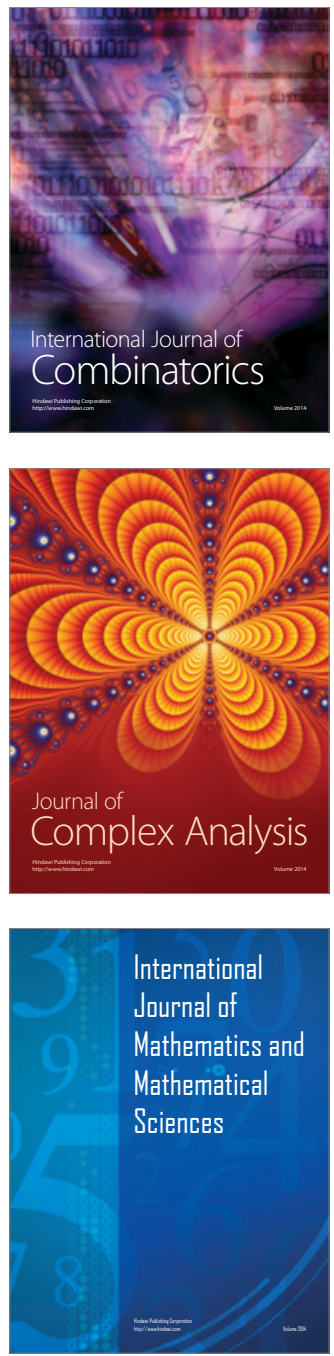
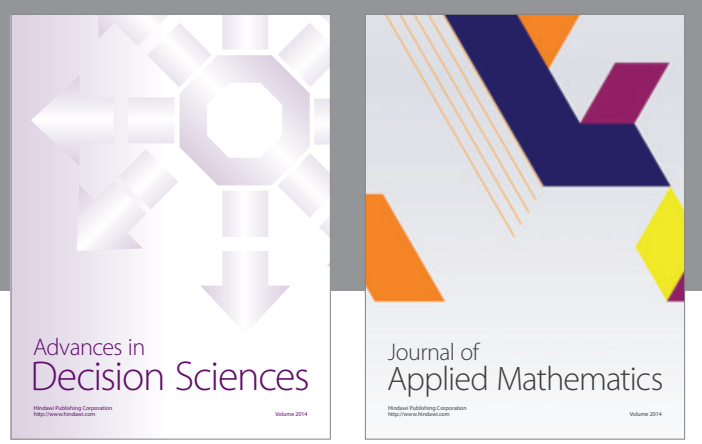

Algebra

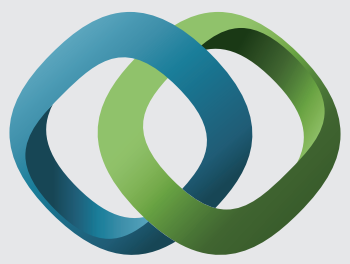

\section{Hindawi}

Submit your manuscripts at

http://www.hindawi.com
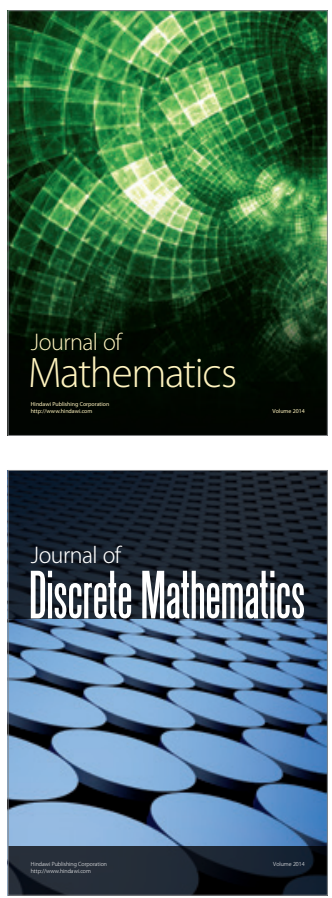

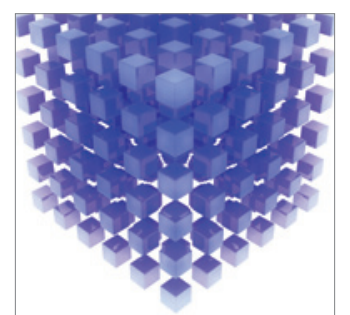

Mathematical Problems in Engineering
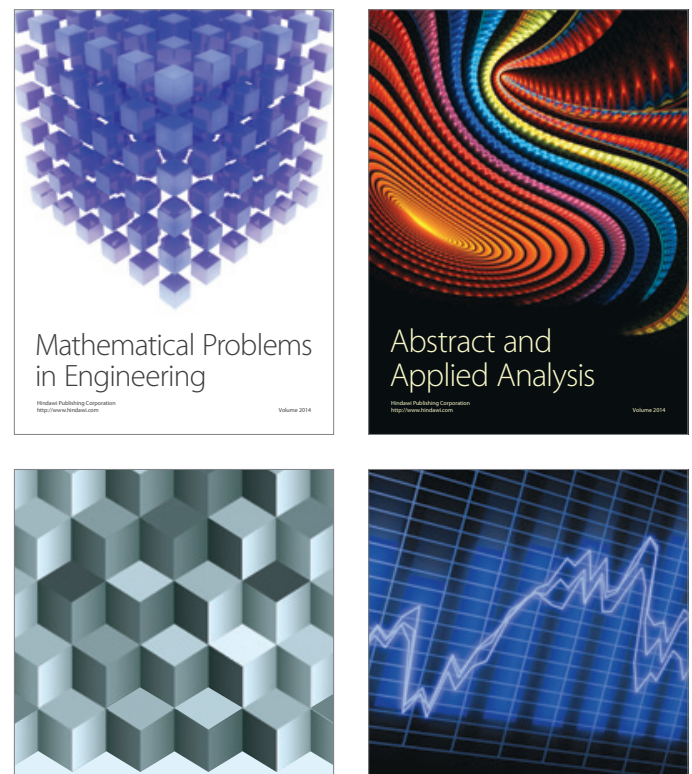

Journal of

Function Spaces

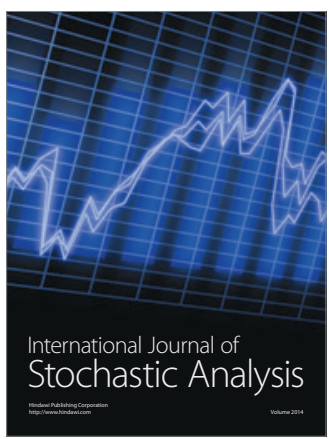

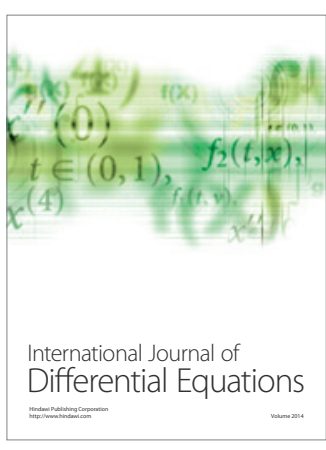
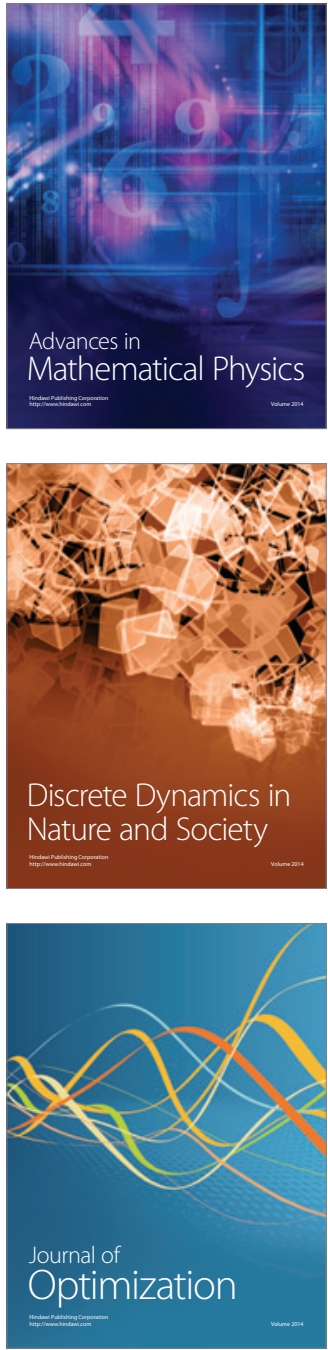